Pure and Applied Mathematics Quarterly

Volume 9, Number 3

$387-416,2013$

\title{
Nonlinearity, Proper Actions and Equivariant Stable Cohomotopy
}

\author{
Noé Bárcenas Torres
}

\begin{abstract}
We extend the classical definition of equivariant cohomotopy theory to the setting of proper actions of Lie groups with analytical methods. We prove the correspondence with previous constructions by constructing an index.
\end{abstract}

Keywords: Equivariant Cohomotopy, Proper Actions, Nonlinear Analysis, Segal Conjecture.

\section{INTRODUCTION.}

Equivariant cohomotopy is an important invariant in algebraic Topology of spaces with actions of compact Lie groups.

In this work, we give a definition of equivariant cohomotopy for spaces with actions of non compact lie groups in terms of certain nonlinear perturbations of Fredholm morphisms of Hilbert bundles over a given proper $G$-CW complex.

The use of analytical methods is due to the fact that the technical difficulties involved in the proof of excision for equivariant cohomology theories, where the equivariance group is neither discrete, nor compact Lie cannot be tackled with constructions using finite dimensional $G$-vector bundles or finite dimensional representations [22], [8].

We prove (Theorem 6.6) that our invariants generalize previous definitions, such as that of W. Lück [14] in the context of proper actions of discrete groups on finite $G$-CW complexes. The proof has its roots in methods employed in

Received October 8, 2010.

Subject Classification: 55P91. Secondary: 2205,47414. 
nonlinear analysis [25], [7], [17]. But is motivated and impregnated by ideas, methods and constructions concerning gauge theoretical invariants in [3].

We illustrate the applications of our methods by several examples. We comment the potential utility of this approach by generalizing a gauge theoretical invariant of 4-dimensional smooth manifolds, due to Bauer and Furuta to allow proper actions of Lie groups on four-manifolds. Finally, we introduce a Burnside ring for non-compact Lie groups in operator theoretical terms .

This paper is organized as follows: in section 1, a review of equivariant cohomotopy for compact Lie groups is given. Preliminaries on Hilbert bundles and Fredholm morphisms between them are given in section 2. Section 3 defines the analytical objects which define equivariant cohomotopy groups for non compact group of symmetries, and examples are considered in section 4 . The properties of equivariant cohomotopy are discussed in 5 , the relation to previous work is stablished in section 6 via a construction of a natural isomorphism, the parametrized Schwarz index. In the last section 7, a Burnside ring for noncompact Lie groups is introduced, and extensions of the Segal conjecture are discussed in this new setting.

0.1. Aknowledgments. The author would like to thank the Mexican Council for Science and Technology, CONACYT for the grant 196868. The main results of this note developed from the corresponding dissertation, defended at the Westfälische Wilhelms-Universität Münster. This work was also supported by the SFB 878 at the University of Münster, Wolfgang Lück's Leibnizpreis, and the Hausdorff Center for Mathematics in Bonn.

\section{Review of Equivariant Cohomotopy for compact Lie Groups.}

In the case of compact Lie groups equivariant cohomotopy is an $R O(G)$-graded equivariant cohomology theory, in the sense of [18].

Definition 1.1. Let $G$ be a compact Lie group and $X$ a $G$-CW complex. For any representation $W$, form the one-point compactification $S^{W}$ and define the set of continuous and pointed maps $\Omega^{W} S^{W}=\operatorname{Map}\left(S^{W}, S^{W}\right)$, where $G$ acts by conjugation. The equivariant cohomotopy group in degree $V=V_{1}-V_{2}$, where $V_{i}$ are finite dimensional real representations, is defined to be the abelian 
Nonlinearity, Proper Actions and Equivariant Stable Cohomotopy 389

group constructed as the colimit of the system of homotopy classes of pointed, equivariant maps

$$
\pi_{G}^{V}(X)=\operatorname{colim}_{W}\left[S^{V_{1}} \wedge X_{+}, \Omega^{W} S^{W \oplus V_{2}}\right]_{G}
$$

where the system runs along a complete $G$-universe, that is a Hilbert space containing as subspaces all irreducible representations, where the trivial representation appears infinitely often.

Finite dimensional representations are involved in this definition in a crucial way and this is the main handicap to extend equivariant cohomotopy to more general settings. In fact, pathological examples from group theory [20] provide finitely generated, discrete groups for which all finite dimensional representations over $\mathbb{R}$, or $\mathbb{C}$ are trivial. Precisely:

Example 1.2. Let $G$ be a finitely presented group. $G$ is said to be residually finite if for every element $g \neq 1$, there exists a homomorphism $\varphi$ to a finite group mapping $g$ to an element different from 1 . The maximal residually finite quotient $G_{m r f}$ of $G$ is the quotient by the normal subgroup consisting of the intersection of all subgroups of finite index. In symbols:

$$
G_{m r f}=\frac{G}{\cap(G: H)<\infty H}
$$

This is a residually finite group, characterized by the property that every group homomorphism to a residually finite group factorizes trough the quotient map. Recall [16], that if $G$ is a finitely generated subgroup of $G l_{n}(F)$ for some field, then $G$ is residually finite. This means that in this situation, every finite dimensional representation of $G$ is induced from one of $G_{m r f}$. An example of Olshanskii, [20] gives for every prime $p>10^{75}$ a finitely generated, infinite group all of whose proper subgroups are finite of order $p$. That means that $G$ does not contain proper subgroups of finite index, hence $G_{m r f}=\{e\}$ and consequently every finite dimensional representation of $G$ is trivial.

\section{Preliminaries on Hilbert Bundles and Fredholm Morphisms}

We recall first some basic definitions and technical facts of equivariant Topology. 
Definition 2.1. Let $G$ be a second countable, Hausdorff locally compact group. Let $X$ be a second countable, locally compact Hausdorff space. Recall that a $G$-space is proper if the map

$$
\begin{gathered}
G \times X \underset{\theta_{X}}{\rightarrow} X \times X \\
(g, x) \mapsto(x, g x)
\end{gathered}
$$

is proper.

Remark 2.2. In the case of Lie groups, a proper action amounts to the fact that all isotropy subgroups are compact and that a local triviality condition, coded in the Slice Theorem is satisfied [21]. Specializing to Lie groups acting properly on $G$-CW complexes, (see the definition below), these conditions boil down to the fact that all stabilizers are compact [13], Theorem 1.23. In particular for an action of a discrete group $G$ on a $G$-CW complex, a proper action reduces to the finiteness of all stabilizer groups.

Definition 2.3. Recall that a $G$-CW complex structure on the pair $(X, A)$ consists of a filtration of the $G$-space $X=\cup_{-1 \leq n} X_{n}$ with $X_{1}=\emptyset, X_{0}=A$ where every space is inductively obtained from the previous one by attaching cells in pushout diagrams

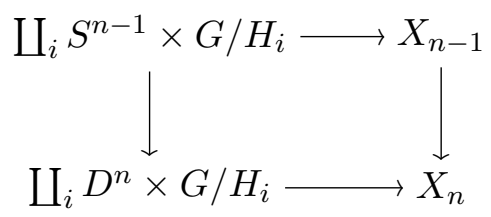

We say that a proper $G$-CW complex is finite if it consists of a finite number of cells $G / H \times D^{n}$.

The following result enumerates some facts which will be needed in the following, which are proven in chapter one of [13]:

Proposition 2.4. Let $(X, A)$ be a proper $G-C W$ pair

(i) The inclusion $A \rightarrow X$ is a closed cofibration.

(ii) $A$ is a neighborhood $G$-deformation retract, in the sense that there exists a neighborhood $A \subset U$, of which $A$ is a $G$-equivariant deformation retract. The neighborhood can be chosen to be closed or open.

We recall the notion of the classifiying space for proper actions: 
Nonlinearity, Proper Actions and Equivariant Stable Cohomotopy 391

Definition 2.5. A model for the classifying space for proper actions is a $G$-CW complex $\underline{\mathrm{E}} G$ with the following properties:

- All isotropy groups are compact

- For any proper $G$-CW complex $X$ there exists up to $G$-homotopy a unique $G$-map $X \rightarrow \underline{\mathrm{E}} G$

The classifiying space for proper actions always exists, is unique up to $G$ homotopy and admits several models. The following list contains some examples. We remit to [15] for further discussion.

- If $G$ is a compact group, then the singleton space is a model for $\underline{\mathrm{E}} G$.

- Let $G$ be a group acting properly and cocompactly on a Cat(0) space $X$. Then $X$ is a model for $\underline{\mathrm{E}} G$.

- Let $G$ be a Coxeter group. The Davis complex is a model for $\underline{\mathrm{E}} G$.

- Let $G$ be a mapping class group of a surface. The Teichmüller space is a model for $\underline{E} G$.

We begin by describing cocycles for equivariant cohomotopy. They are defined in terms of certain nonlinear operators on real $G$-Hilbert bundles, so we briefly recall some well-known facts on then and their morphisms. A comprehensive treatment of Hilbert bundles and their linear morphisms is given in the book [22]. For matters related to real $C^{*}$-algebras we refer to the text [24], in particular for connections with Kasparov $K K$-theory.

Definition 2.6. Let $X$ be a locally compact, Hausdorff proper $G$-space. A Banach bundle over $X$ is a locally trivial fiber bundle $E$ with fiber modeled on a Banach space $H$, whose structure group is the set of all isometric linear bijections of $H$ with the strong topology.

If $H$ is a (real) Hilbert space, we will speak of a Hilbert bundle. A $G$-Hilbert bundle is a Hilbert bundle $p: E \rightarrow X$ endowed with a continuous action of the locally compact group $G$ in the total space. The map $p$-called the projection- is assumed to be $G$-equivariant and the action on the total space is given by linear isometric bijections.

Definition 2.7 (Linear Morphism). Let $E$ and $F$ be Hilbert bundles over $X$. A linear morphism from $E$ to $F$ is an equivariant, continuous function $t: E \rightarrow F$ covering the identity on $X$ consisting of bounded operators on fibers and in 
adition, for every point $x \in X$, there exist local trivializations $a:\left.E\right|_{U} \rightarrow U \times E_{x}$, $b:\left.F\right|_{U} \rightarrow U \times F_{x}$ such that $b t a^{-1}: U \times E_{x} \rightarrow U \times F_{x}$ is given by an expresion $(y, x) \mapsto(y, \psi(x))$ for a norm continuous map $\psi: U \rightarrow L\left(E_{x}, F_{x}\right)$ to the bounded, adjoinable linear maps between the fiber over $x$. The support of a morphism $t$ is the set $\{x \in X \mid t x \neq 0\}$.

Definition 2.8. Denote by $I$ the unit interval. Two linear morphisms $l_{0}, l_{1}$ : $E \rightarrow F$ are homotopic if there exists a linear morphism $H: E \times I \rightarrow F$ which covers the projection $X \times I \rightarrow X$ and restricts to 0,1 to the morphisms $l_{0}, l_{1}$.

Definition 2.9 (Compact linear morphism). A linear morphism $t: E \rightarrow F$ is a compact morphism if it is fiberwise compact in the usual sense (that is, for every $x, t_{x} \cdot E_{x} \rightarrow F_{x}$ maps bounded sets to relatively compact sets).

Definition 2.10 (Fredholm morphism). A linear morphism $t: E \rightarrow F$ is said to be Fredholm if there exists a morphism $s: F \rightarrow E$ such that $s t-1$ and $t s-1$ are compact morphisms with compact supports. A Fredholm morphism is said to be essentially unitary if one can take $t=s^{*}$ in the definition. We recall the existence of $G$-invariant riemannian metrics on vector bundles over proper $G$-spaces, which is proved for instance in [21] as consecuence of the slice theorem.

Remark 2.11. $\quad$ (i) Notice that we choose the structure group of our bundles to be the isometric bijections with the strong operator topology. Other choices, like the weak *-topology would not give enough morphisms, as pointed out by Phillips in [22], chapter 9.

(ii) Notice that we forced the existence of adjoints for our morphisms. This is technically convenient. We also assume that adjoint operators exist and are always continuous in the operator norm, and the same for morphisms of Hilbert bundles.

We now ennumerate a collection of facts on linear morphisms and $G$-Hilbert bundles which we will use later.

Proposition 2.12 (Proper stabilization theorem). Let $E$ be a G-Hilbert bundle over a proper $G$-space $X$. Denote by $\mathcal{H}$ the numerable Hilbert space consisting of the numerable sum of the space of square integrable functions in $G$, in symbols $\mathcal{H}=\oplus_{n=1}^{\infty} L^{2}(G)$. Let $\mathcal{H} \times X$ be the associated trivial Hilbert $G$-bundle over $X$. There exists an equivariant linear isomorphism of $G$-Hilbert Bundles

$$
E \oplus \mathcal{H} \times X \cong \mathcal{H} \times X
$$


Nonlinearity, Proper Actions and Equivariant Stable Cohomotopy 393

Proof. Theorem 2.9, p. 29 of [22], Theorem 2.1.4, p. 58 of [24] in the real case. We point out that Phillips realizes this isomorphism as an adjoinable morphism between the $G$ - $C_{0}(X)$ - Hilbert modules $\Gamma(E) \otimes_{C_{0}(X)} \Gamma(\mathcal{H} \times X)$ and $\Gamma(X \times \mathcal{H}) \otimes_{C_{0}(X)} C_{0}(X)$. The identification of such a morphism with an isomorphism of $G$-Hilbert bundles is consequence of lemma 1.9 in [22].

Next, we modify Phillip's definition of complex equivariant $K$-theory for proper actions of locally compact groups, [22] to allow real cocycles. The main reference for technical isssues concernig the passage to real $K$-theory is [24].

Definition 2.13. The real equivariant $K$-theory of the proper and finite $G$-CW complex $X K O(X)_{G}^{0}$ is represented by cocycles $(E, F, l)$, where $E$ and $F$ are real $G$-Hilbert bundles and $l: E \rightarrow F$ is a fiberwise linear real Fredholm morphism. A cocycle is said to be trivial if $l$ is fibrewise unitary. Two cocycles $\left(E_{i}, F_{i}, l_{i}\right)_{i=0,1}$ are equivalent if there exists a trivial cocycle $\tau$ such that $l_{0} \oplus \tau=l_{1} \oplus \tau$ is homotopic to a trivial morphism.

We now enumerate two consequences of the proper stabilization theorem, which are fundamental for veryfing excision in Phillips' construction of equivariant $K$ theory.

Proposition 2.14. Let $i: U \rightarrow X$ be the inclusion of a $G$-invariant, open subset of $X$. Let $(E, F, t)$ be a linear cocycle over $U$ such that $t$ is fibrewise bounded and has a bounded Fredholm inverse. Then, there exists a linear cocycle $(X \times \mathcal{H}, X \times \mathcal{H}, r)$ such that the classes $i^{*}(r)$ and $t$ agree after adding a unitary linear cocycle.

Proof. Proposition 5.9, p. 74 in [22]. We recall that the constructed classes agree after application of the proper stabilization theorem, for the real modification, see Theorem 2.1.4 p.58 in [24] .

Proposition 2.15. Let $X$ be a proper $G-C W$ complex and $\varphi: E \rightarrow E$ be a Fredholm morphism defined over the space $X \times I$. Denote by $\varphi_{0}: E_{0} \rightarrow E_{0}$ the restriction to $X \times\{0\}$. Then: 
(i) There exists a unitary cocycle $\rho$ between $(E, E, \varphi)$ and $\left(E_{0}, E_{0}, \varphi_{0}\right)$. Moreover, the isomorphism can be taken to be unitary over a fixed, invariant subspace $A \subset X$.

(ii) Let $A \subset X$ be a G-subcomplex and $l:\left.\left.F\right|_{A} \rightarrow E\right|_{A}$ be a bounded morphism. Then, there exists a linear cocycle $\left(l^{\prime}, E, F\right)$, defined over $X$ such that $i^{*}(l)$ and $l$ are equivalent .

(iii) Let $A \subset X$ be a $G$-invariant closed subset and $U \subset X$ an open neighborhood of which $A$ is a deformation retract. Suppose that $(U \times K, U \times K, l)$ is an essentially unitary cocycle over $U$, where $K$ is a strong continuous unitary $G$-representation in a Hilbert space. Then, there exists an essentially unitary cocycle $(X \times H, X \times H, F)$ such that $i^{*}(F)=l$.

Proof. (i) As the involved bundles are locally trivial $E$ carries the weak topology with respect to the set $p^{-1}\left(X_{i}\right)$, where $X_{i}$ is an element of the $C W$ - filtration in the basis (cfr. lemma 1.26 in [13]). Hence, the statement reduces to the case where $(X, A)=\left(G / H \times D^{n}, G / H \times S^{n-1}\right)$ and $E$ has the form $G \times \mathcal{H}$, for a given strong- norm continuous and unitary representation of the compact lie group $H$ in a separable real Hilbert space $\mathcal{H}$. Let $\mathcal{U}_{c}(\mathcal{H})$ be the subspace of the $H$-equivariant, unitary operators $u$ in $\mathcal{H}$, for which the conjugation with an arbitrary element $h^{-1} u h$ is a continuous operator (recall that this is a contractible space after results of Segal[1], Apendix 3 for the compact Lie case). Giving an isomorphism $\rho$ as described above amounts to give a map $\rho \in \operatorname{Map}\left(D^{n} \times I, \mathcal{U}_{c}(\mathcal{H})\right)$ which is the identity on $G / H \times S^{n-1} \times\{0\}$. There is no obstruction for doing this because the inclusion $G / H \times S^{n-1} \rightarrow G / H \times D^{n}$ is a cofibration and $\mathcal{U}_{c}(\mathcal{H})$ is contractible.

(ii) It follows from the reduction to a cell as above and the fact that for a compact group $H$, the space of $H$-equivariant compact operators is contractible.

(iii) It follows from the restriction to a cell as above and the contractibility of the equivariant unitary group of a Hilbert representation for a compact group. 
Nonlinearity, Proper Actions and Equivariant Stable Cohomotopy 395

\section{COCYCles For EQUivariant StABle COHOMOTOPY}

The second ingredient for our construction of cocycles for stable cohomotopy are some basic notions of nonlinear functional analysis. References to this topic are the books [4] and [6]. Applications to differential equations can be found in [12], [17]. From this section on, all groups are assumed to be Lie, actions are assumed to be proper and all spaces are finite $G$-CW complexes.

Definition 3.1. Let $X$ be a locally compact, proper $G$-CW complex and let $G$ be a locally compact group.

A cocycle for the equivariant cohomotopy theory of $X, \Pi_{G}^{[l]}(X)$ is a four-tuple $(E, F, l, c)$ where

- $E, F$ are real $G$ - Hilbert bundles over $X$, with a linear, real Fredholm morphism $l: E \rightarrow F$.

- A map $c: E \rightarrow F$, lifting the identity on $X$ and possibly nonlinear on each fibre, for which there exist local trivializations $a:\left.E\right|_{U} \rightarrow U \times E_{x}$, $b:\left.F\right|_{U} \rightarrow U \times F_{x}$ such that $b t a^{-1}: U \times E_{x} \rightarrow U \times F_{x}$ is given by a continuous expresion $(y, x) \mapsto(y, k(x))$ consisting of possibly nonlinear, compact and continuous maps, in a way that $l+c$ extends to a map between one-point compactification bundles.

Two cocycles $(E, F, l, c)$ and $\left(E^{\prime}, F^{\prime}, l^{\prime}, c^{\prime}\right)$ are equivalent if there is a linear, unitary cocycle $\left(H, H^{\prime}, u\right)$ such that $(E \oplus H, F \oplus H, l \oplus u)$ and $\left(E^{\prime} \oplus H^{\prime}, F^{\prime} \oplus H^{\prime} l^{\prime} \oplus u\right)$ are unitary equivalent as linear cocycles, by an isomorphism which intertwines $c$ and $c^{\prime}$.

Two cocycles $(E, F, l, c)$ and $\left(E, F, l, c^{\prime}\right)$ are homotopic if there exists a homotopy $H: S^{E} \times I \rightarrow S^{F}$, covering the projection $X \times I \rightarrow X$, pointed over every fiber and relative to $l$. That means, $H$ has the form $l+h$ for a certain compact map $h: S^{E} \times I \rightarrow S^{F}$ such that $\left.H\right|_{0}=l+c,\left.H\right|_{1}=l+c^{\prime}$.

The set $\Pi_{G}^{[l]}(X)$ is called the $G$-equivariant cohomotopy of $X$ in degree $[l]$.

In several applications in analysis, [17], [10], cocycles have a diferent, but equivalent shape:

Remark 3.2 (The "no zeros on the boundary"-picture). Equivariant cohomotopy also can be described as four-tuples $(E, F, t, k)$ where $t: E \rightarrow F$ is a real 
Fredholm morphism between $G$-Hilbert bundles, with a choice of an invariant riemannian metric on $E, k$ is a non necessary linear, compact fibrewise map defined on the unitary disk $D(E)=\{x \in E|| x \mid \leq 1\}$ which is strongly non zero, in the sense that $k x=0$ has no solutions on the boundary of the unit disk $\partial D=\{x \in E|| x \mid=1\}$. Cocycles are said to be equivalent if they agree after adition of a linear, unitary Fredholm morphism. The homotopy condition is defined with homotopies which not only are assumed to be compact, but also strongly non-zero and relative to $l$, that is, a homotopy is a map of the form $l+h$, where $h$ is a compact map defined on $D E \times I$, for which for any $t$, the corresponding map has no zeros on the boundary $\partial D(E)$.

The coincidence of both approaches in the case $X=\{\bullet\}$ with an action of a compact lie group, as for example in [17] follows from the fact that a strongly nonzero compact perturbation gives a map of pairs $(D(E), \partial D(E)) \rightarrow(E, E-\{0\})$, which are equivalent via excision and homotopy equivalence to maps $\left(S^{E}, \infty\right) \rightarrow$ $\left(S^{E}, \infty\right)$, via the intermediary pairs $\left(S^{E}, S^{E}-\operatorname{Int} D(E)\right)$ and $\left(S^{E}, E-\{0\}\right)$, respectively.

The main difference between our definition an the classical one is the fact that our theory is graded by the group $K O(X)_{G}^{0}$, instead of the representation ring. $K O(X)_{G}^{0}$-theoretical graded invariants play a role in [19], [3] and [23].

\section{Examples of Cocycles for equivariant cohomotopy}

I this section we will give some of the most common examples of cocycles in equivariant cohomotopy from the analytical viewpoint. The first four of them are obtained by modifying classical constructions. The last example generalizes an invariant due to Stefan Bauer and Mikio Furuta in Gauge Theory and is not indispensable for the rest of the paper.

Example 4.1 (Collapse map related to the Thom-Pontrjagyn Construction). Let $M$ be a framed, orientable $k$-dimensional manifold with an embedding $M \rightarrow U \subset$ $\mathbb{R}^{n+k}$, where $U$ is a tubular neighborhood. By collapsing the complement of $U$ to infinity, and choosing an homeomorphism of the tubular neighborhood and using the framing to project onto $S^{\mathbb{R}^{n}}$ one constructs a proper map $c: S^{\mathbb{R}^{n+k}} \rightarrow S^{\mathbb{R}^{n}}$. The cocycle $\left(\mathbb{R}^{n+k}, \mathbb{R}^{n}, 0, c\right)$ represents an element in $\Pi_{\{1\}}^{k}(\{*\})$ in our sense. 
Example 4.2 (G-Euclidean Neighborhood Retracts). Slightly more general, consider a compact lie group $G$, and an equivariant euclidean neighborhood retract, that is, a compactly generated space $X$ with an embedding $X \rightarrow V$ into some finite dimensional representation of $G$, and a retraction $r: U \rightarrow X$ of some open invariant subset $U \subset V$. In an analogous situation to the manifold case, Tom Dieck [28], p.188 constructs a collapse map, called the Lefschetz-Dold index

$$
I_{G}(X): S^{V} \rightarrow S^{V}
$$

The element $\left(V, V, 0,\left.I_{G}(X)\right|_{V}\right) \in \Pi_{G}^{0}(\{*\})$ represents a cocycle.

Example 4.3 (Parametrized Fixed Point Situations). Let $B$ be a metric space with an action of a compact lie group $G$. An euclidean neighborhood retract over $B$ is an equivariant, locally trivial fibration $p: E \rightarrow B$ endowed with a fiberwise embedding into a trivial bundle $E \rightarrow V \times B$. A parametrized fixed point situation is a diagram of the form

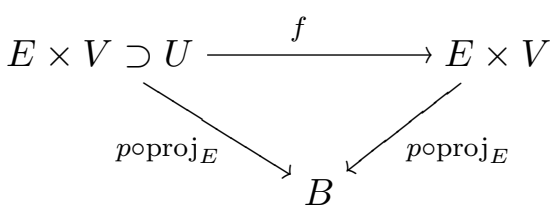

where $f$ is a compactly fixed map, in the sense that the restriction of $p$ to the fixed point set $\operatorname{Fix}(f)=\{(e, y) \in V \mid f(e, y)=(e, 0)\}$ is a proper map onto $B$.

Under these hypotheses there exists a $G$-invariant neighborhood $N$ of $\operatorname{Fix}(f)$ which is relatively compact and contained in $U$, as well as a positive number $\epsilon$ such that $\left|\operatorname{proj}_{V}(f x)-\operatorname{proj}_{V} x\right| \geq \epsilon$ for all $x \in \bar{N}-N$ for some $G$-invariant metric in $V$. There exists a map $c: U \rightarrow S^{U}$ which maps the open ball of radius $\epsilon$ and centre 0 homeomorphically into $U$. Consider the map $k(x)=\left(c\left(\operatorname{proj}_{V} x\right)-\operatorname{proj}_{V} f(x), x\right)$ defined on $U$, which is proper. The element $(B \times V, B \times V, 0, k)$ represents a cocycle in $\Pi_{G}^{0}(B)$.

Parametrized equivariant fixed point theory was developed in detail in [30] in the context of actions of compact lie groups, generalizing the foundational work of Dold [7].

Example 4.4 (Nonlinear Elliptic Operators with Symmetry). Let $\Omega \subset V$ be a $G$-invariant, relatively compact, open domain with smooth boundary inside some real representation $V$ of the compact lie group $G$. Consider the Hölder spaces 
$C^{k+\mu}(\bar{\Omega}, V)=C^{k}(\bar{\Omega}, \mathbb{R}) \otimes V$ for $\mu \in(0,1)$ and an elliptic linear operator of order $\mathrm{m}:$

$$
P=\sum_{|\alpha| \leq m} A_{\alpha}(X) D^{\alpha}
$$

where the matrix-valued functions $A_{\alpha}: \bar{\Omega} \rightarrow \operatorname{Hom}_{\mathbb{R}}(V, V)$ are $G$-equivariant. Given a set of well-posed, $G$-equivariant boundary conditions $B$, and any $\mu \in$ $(0,1)$ Marzantowicz [17] constructs a $G$-equivariant, Fredholm operator between Banach spaces

$$
P: C_{P, B}^{k+m+\mu}(V)=\left\{x \in C^{k+m+\mu}(\bar{\Omega}, V) \mid B(x)=0\right\} \rightarrow C^{k+\mu}(\bar{\Omega}, V)
$$

defined in a subspace $C_{P, B}^{k+m+\mu}(V)$ of the Hölder space satisfying the boundary conditions.

In an analogous situation, considering the elliptic operator in the Hilbert space $H^{s, 2}(\Omega, \mathbb{R})$ of all functions having formal derivatives in $L^{2}$ up to a certain order $s$ gives a $G$-Hilbert space $H^{s, 2}(\bar{\Omega}, V)=H^{s}(\bar{\Omega}, \mathbb{R}) \otimes V$, as well as a Fredholm operator defined on the space where the boundary conditions $B$ are satisfied, which we also denote by $P_{B}: H_{B}^{s+m, 2}(\bar{\Omega}, V) \rightarrow H_{B}^{s, 2}(\bar{\Omega}, V)$

For any strongly nonzero, nonlinear equivariant function defined on the radius one disk $\Phi: D_{1}\left(H_{B}^{s+m}(\bar{\Omega}),\right) \rightarrow H^{s}(\bar{\Omega}, V)$, the cocycle

$$
\left(H_{B}^{s+m}(\bar{\Omega}, V), H^{s}(\bar{\Omega}, V), P_{B}, \Phi\right)
$$

represents an element in the "no-zeros on the boundary"-picture of

$$
\Pi_{G}^{\mathrm{ker}\left(\mathrm{P}_{\mathrm{B}}\right)-\operatorname{coker}\left(\mathrm{P}_{\mathrm{B}}\right)}(\{*\})
$$

See [17], definition 1.2 for the definition of an explicit isomorphism (the equivariant Leray-Schauder degree) of an specific kind of these objects to the equivariant stems of a compact Lie group. This is the equivariant version of the main result of the foundational work of Albert Schwarz, [25]. See also [12] for applications of related constructions to Hopf bifurcation problems.

We illustrate now an example where our constructions in terms of perturbation of Fredholm morphisms appear in a natural way. This approach was used by Stefan Bauer [3] and Mikio Furuta, see also [19] and [26].

We recall briefly that in the context of smooth, riemannian, oriented 4-dimensional manifolds, the existence of a $\operatorname{Spin}^{c}(4)$-structure is always guaranteed. This amounts 
Nonlinearity, Proper Actions and Equivariant Stable Cohomotopy 399

to a map from a $\operatorname{Spin}^{c}(4)$ principal bundle $Q$ together with a bundle map $Q \rightarrow P$ to the frame bundle of the tangential bundle. The identification of the group $\operatorname{Spin}^{c}(4)$ with the sugroup $\left\{u_{+}, u_{-} \mid u_{-}, u_{+} \in \mathrm{U}(2), \operatorname{det}\left(u_{+}\right)=\operatorname{det}\left(u_{-}\right)\right\}$allows to define positive, respectively, negative spinor bundles $S^{+}, S^{-}=Q \underset{\rho^{+,-}}{\times} \mathbb{C}^{2}$, where $\rho_{+,-}: \operatorname{Spin}^{c} \rightarrow \mathrm{U}(2)$ are the respective projections. Using quaternionic multiplication, it is posible to furnish $S:=S^{+} \oplus S^{-}$with the structure of a module over the Clifford algebra of the cotangential bundle $T^{*}(X) \times S^{+,-} \rightarrow S^{-,+}$. Clifford identities give a linear map $\rho: \Lambda^{2} \rightarrow \operatorname{End}_{C}\left(S^{+}\right)$whose kernel is the bundle of anti-selfdual 2-forms and whose image is the bundle of trace free skew hermitian endomorphisms. For any $\operatorname{spin}^{c}$-connection $A$, define the associated Dirac operator $D$ as the composition $\Gamma\left(S^{+}\right) \underset{\nabla_{A+a}}{\rightarrow} \Gamma\left(S^{+}\right) \otimes \Lambda^{1}\left(T^{*} M\right) \stackrel{\gamma}{\rightarrow} \Gamma\left(S^{-}\right)$, where $\gamma$ denotes Clifford multiplication.

The monopole map $\tilde{\mu}$ is defined for four-tuples $(A, \phi, a, f)$ of a $\operatorname{Spin}^{c}$ connection $A$, a positive spinor $\phi$, a 1-form $a$ and a locally constant function $f$ on $M$ as

$$
\begin{gathered}
\mu: \text { Conn } \times \Gamma\left(S^{+}\right) \oplus \Omega^{1}(M) \oplus H^{0}(M) \rightarrow \\
\operatorname{Conn} \times \Gamma\left(S^{-}\right) \oplus \Omega^{+}(M) \oplus \Omega^{0}(M) \oplus H^{1}(M) \\
(A, \phi, a, f) \mapsto\left(A, D_{A+a} \phi, F_{A+a}^{+}-\sigma(\phi), d^{*}(a)+f, a_{\text {harm }}\right)
\end{gathered}
$$

where $\sigma$ is the trace free endomorphism $(-i)\left(\phi \otimes \phi^{*}\right)-\frac{1}{2}|\phi|^{2}$ id, and $F^{+}$ denotes the self-dual part of the curvature. Given a point in $M$, the based gauge group $\mathcal{G}_{x}$ is the kernel of the evaluation map at $x . \operatorname{map}\left(X, S^{1}\right) \rightarrow S^{1}$. The subspace $A+\operatorname{ker}(d)$ is invariant under the free action of the based gauge group. The quotient is isomorphic to the Picard torus, $\mathfrak{P i c}(X)=H^{1}(X, \mathbb{R}) / H^{1}(X, \mathbb{Z})$. Let $\mathcal{A}$ and $\mathcal{B}$ be the quotients

$$
a+\operatorname{ker} d \times \Gamma\left(S^{+}\right) \oplus \Omega^{1}(X) \oplus H^{0}(X, \mathbb{R}) / G_{x}
$$

respectively

$$
a+\operatorname{ker} d \times \Gamma\left(S^{-}\right) \oplus \Omega^{+}(X) \oplus \Omega^{0}(X, \mathbb{R}) \oplus H^{1}(X, \mathbb{R}) / G_{x}
$$

the quotient map $\mu: \tilde{\mu} / G_{x}: \mathcal{A} \rightarrow \mathcal{B}$ has by definition a presentation as a fibrewise compact perturbation of a Fredholm operator. It is proper after a result of Bauer 
and Furuta, [3], which essentially uses estimates determined by the Weitzenböck formula. This gives rise to a cocycle. $\mathcal{A}, \mathcal{B}, D_{A}+d+d^{*}, c=F_{A}^{+}+a \cdot \phi+\sigma(\phi)$, where $\sigma$ is the selfdual trace free endomorphism $\phi \mapsto\left(-i \phi \otimes \phi^{*}-\frac{1}{2}|\phi|\right)$.

Example 4.5. Suppose $G$ is a (possibly noncompact) Lie group acting properly and cocompactly on the smooth $\operatorname{Spin}^{c}$ - manifold $M$. assume furthermore, that the group preserves the orientation and by means of isomorphisms of complex Spin $^{c}$ structures, and respecting the $\operatorname{Spin}^{c}$-connection. As the action is proper, it can be assumed that $G$ preserves the metric. Let $\mathbb{G}$ be the group of pairs $(\varphi, u)$, where $\varphi$ is a $G$-equivariant diffeomorphism which preserves both the metric and the orientation and $u: f^{*}\left(\sigma_{M}\right) \rightarrow \sigma_{M}$ is an isomorphism of the $\operatorname{Spin}^{c}$ principal

bundle. In particular, this gives a description of $\mathbb{G}$ in the middle of the following exact sequence

$$
1 \rightarrow S^{1} \rightarrow \mathbb{G} \rightarrow G \rightarrow 1
$$

In this situation, the class of $\mu$ is denoted by

$$
m_{\mathbb{G}}\left(X, \sigma_{X}\right) \in \Pi_{G}^{\operatorname{ind}(\lambda)}(\mathfrak{P i c}(X))
$$

and we call it the generalized Bauer-Furuta invariant. The restriction map

$$
\operatorname{res}_{\mathbb{G}}^{S^{1}}: \Pi_{\mathbb{G}}^{\operatorname{ind}(l)}(\mathfrak{P i c}(X)) \rightarrow \Pi_{S^{1}}^{\operatorname{ind}(l)}(\mathfrak{P i c}(X)) \cong \Pi_{S^{1}}^{\operatorname{ind}(l)}(\mathfrak{P i c}(X))
$$

which will be constructed in the following section maps $m_{\mathbb{G}}$ to the $S^{1}$-equivariant cohomotopical Bauer-Furuta invariant defined in [3].

\section{Cohomological Properties of Equivariant Cohomotopy for PROPER ACTIONS}

We describe now an additive structure in equivariant cohomotopy theory.

Let $\left(E_{0}, F_{0}, l_{0}, c_{0}\right)$ and $\left(E_{1}, F_{1}, l_{1}, c_{1}\right)$ be cocycles in the equivariant cohomotopy theory of a given degree $[l]$.

Let us suppose without loss of generality that we have representatives of the form $\left(E_{0}, F_{0}, l, c_{0}\right)$ and $\left(E_{0}, F_{0}, l, c_{1}\right)$.

Let $X \times \mathbb{R} \rightarrow X \times \mathbb{R}$ be the trivial bundle and. Denote by $S^{\mathbb{R}}$ the one point compactification bundle. Define the pinching map $\nabla: S^{\mathbb{R}} \rightarrow S^{\mathbb{R}} \vee S^{\mathbb{R}} \approx$ 
Nonlinearity, Proper Actions and Equivariant Stable Cohomotopy 401

$$
\begin{array}{r}
\left(S^{\infty}, 0\right) \vee\left(S^{-\infty}, 0\right) \\
\nabla(x)=\left\{\begin{array}{l}
\ln (-x)(x \in-\infty,-1] \\
-\ln (-x) x \in(-1,0] \\
\infty x=0 \\
\ln (x) x \in(0,1] \\
-\ln (x) x \in(1, \infty) \\
-\infty x=\infty
\end{array}\right.
\end{array}
$$

The sum of two cocycles is represented by the cocycle $\left(E_{0} \oplus \mathbb{R}, F_{0} \oplus \mathbb{R}, l \oplus \mathrm{id}, c\right)$, where $l \oplus \mathrm{id}+c: S^{E_{0} \oplus \mathbb{R}} \rightarrow S^{F_{0} \oplus \mathbb{R}}$ is given as the composition

$$
\begin{aligned}
S^{E_{0}} \wedge_{X} S^{\mathbb{R}} \stackrel{\mathrm{id} \wedge_{X} \nabla}{\longrightarrow} S^{E_{0}} \wedge_{X} S^{\mathbb{R}} \vee_{X} S^{\mathbb{R}} \stackrel{\approx}{\rightarrow} \\
S^{E_{0} \oplus \mathbb{R}} \vee_{X} S^{E_{0} \oplus \mathbb{R}} \stackrel{\left(l \oplus \mathrm{id}+c_{0}\right) \wedge_{X}\left(l \oplus \mathrm{id}+c_{1}\right)}{\longrightarrow} S^{F_{0} \oplus \mathbb{R}}
\end{aligned}
$$

The zero element is represented by a cocycle $(E, F, l, c)$ such that $l+c$ extends to a map sending constantly $S^{E}$ to the point at infinity.

The inverse of an element $(E, F, l, c)$ is represented by the element $(E \oplus \mathbb{R}, F \oplus$ $\mathbb{R}, l \oplus i d, c \oplus-i d)$. We have the following result:

Proposition 5.1. The operations described above define an abelian group structure in equivariant cohomotopy.

There is a relative version for pairs $(X, A)$ of proper $G$-CW complexes. An element in $\Pi_{G}^{[l]}(X, A)$ is represented by a compact perturbation of a fibrewise perturbation of a Fredholm morphism $l+c: E \rightarrow F$, which extends to the one-point compactification bundles being constant over the subspace $A$, with the value at infinity. Notice that this is consistent with the usual identification of $X$ with the pair $(X, \emptyset)$.

We construct a multiplicative structure on the equivariant cohomotopy theory:

$$
\cup: \Pi_{G}^{\left[l_{1}\right]}\left(X, A_{1}\right) \times \Pi_{G}^{\left[1_{2}\right]}\left(X, A_{2}\right) \rightarrow \Pi_{G}^{\left[l_{1}+l_{2}\right]}\left(X, A_{1} \cup A_{2}\right)
$$

Consider for this representing elements $u_{i}=l_{i}+c_{i} \in \Pi_{G}^{\left[l_{i}\right]}\left(X, A_{i}\right)$ for $i \in\{1,2\}$, where $c_{i}$ is a compact map accepting fibrewise an extension to the one-point compactification, constant over $A_{i}$ with the value at infinity. $u_{1} \cup u_{2}$ is the cocycle defined as $\left(E_{1} \oplus E_{2}, \varphi_{1} \oplus \varphi_{2}, C\right)$ where the map $C$ is such that $C$ : $\left(e_{1}, e_{2}\right) \mapsto\left(c_{1}\left(e_{1}\right), c_{2}\left(e_{2}\right)\right)$. Notice that this map allows an extension to the onepoint compactification. 
We investigate now the cohomological behaviour of equivariant cohomotopy:

Proposition 5.2 (Functoriality). Let $f:(X, A) \rightarrow(Y, B)$ be a $G$-map between finite $G$-CW complexes. Then $f$ induces a group homomorphism

$$
\Pi_{G}^{[l]}(Y, B) \rightarrow \Pi_{G}^{f^{*}[l]}(X, A)
$$

Proof. Follows from the naturality of the pullback construction for Hilbert bundles, Fredholm morphisms and compact perturbations.

Proposition 5.3. Let $(X, A)$ be a proper $G-C W$ pair. There exists a natural sequence

$$
\Pi_{G}^{[l]}(X, A) \stackrel{\rho^{*}}{\rightarrow} \Pi_{G}^{[l]}(X) \stackrel{i^{*}}{\rightarrow} \Pi_{G}^{[l]}(A)
$$

which is exact in the middle, where $\rho$ and $i$ denote the inclusion of $A$ into $X$ and $X$ into $(X, A)$, respectively.

Proof. That $i^{*} \circ \rho^{*}=0$ is clear form the definitions. Let now $l+c: E \rightarrow F$ be a cocycle for which $i^{*}([l+c])$ is compactly homotopic to the trivial morphism over A.

In view of proposition 2.12, we can choose a representative (which we denote by the same symbols) for which both $E$ and $F$ are the trivial $G$-Hilbert bundle $\mathcal{H} \times X \rightarrow X$ and the extension to one point compactification bundles is constant over $A$ with value $\infty$. Using proposition 2.14 , we can assume up to equivalence that the perturbation of the Fredholm morphism extends to a map $\tilde{l}$ defined between bundles defined over all points of $X$.

Suppose that there is a homotopy $h_{t}: i^{*} S^{E} \times I \rightarrow i^{*} S^{F}$ defined over $A$ which begins with $i^{*}[l+c]$ and ends with a map $l+c$ which sends $S^{E}$ to the base point at infinity. As $X-A$ is built up out of a finite number of equivariant cells, one can argue inductively to extend $h$ to a map $H: S^{E} \rightarrow S^{F}$, defined on all $X$ such that $\left.h\right|_{A \times I}=h . H$ determines a homotopy between certain element $\rho^{*}(\tilde{l}+\tilde{c})$ defined over $X$ and $l+c$.

Proposition 5.4. Let $(X, B)$ be a proper, finite $G-C W$ pair obtained as the pushout with respect to the cellular map $(f, F):\left(X_{0}, A\right) \rightarrow(X, B)$ as in the 
Nonlinearity, Proper Actions and Equivariant Stable Cohomotopy 403

following diagram:

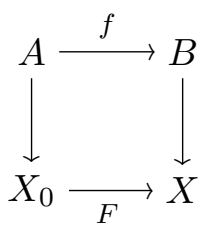

then the $\operatorname{map}(f, F)^{*}: \Pi_{G}^{[l]}(X, B) \rightarrow \Pi_{G}^{[l]}\left(X_{0}, A\right)$ induces a natural isomorphism.

Proof. Let $(E, F, l, c) \in \Pi_{G}^{[l]}\left(X_{0}, A\right)$. Due to propositon 2.14 , it is possible to assume that there exists a linear morphism $\tilde{l}: E \oplus E^{\prime} \rightarrow F \oplus E^{\prime}$ defined over $X$ such that $F^{*}(\tilde{l})$ and $l i$ differ by addition of an unitary cocycle. As $\left.c\right|_{A}=\infty$, it is posible to extend $c$ to a map $\tilde{c}$ defined on $X$, which extends to one point compactification bundles for which $\tilde{l}+\left.\tilde{c}\right|_{B}=\infty$. Then $\left(F^{*}, f^{*}\right): \Pi_{G}^{*}(X, B) \rightarrow$ $\Pi_{G}^{*}\left(X_{0}, A\right)$ sends $\tilde{l}+\tilde{c}$ to $l+c$. This proves surjectivity. To prove injectivity, recall that if $l+h_{t}: E \times I \rightarrow E$ is a nullhomotopy starting with the extension to one point compactification bundles of $\left(F^{*}, f^{*}\right)(l+c)$, ending with the constant $\infty$. As before, as $c$ is constant over $B$ and $\left(F^{*}, f^{*}\right)(c)$ is trivial over $A$, it is possible to extend the map $h_{t}$ to a homotopy $\tilde{h}_{t}$ defined over $X$ which is trivial over $B$ and which begins with $l+c$, and ends with a constant map. This shows that the map is injective.

Proposition 5.5 (Homotopy invariance). Let $f_{0}, f_{1}:(X, A) \rightarrow(Y, B)$ be two $G$ maps of pairs of proper $G$-CW complexes. If they are homotopic, then $\Pi_{G}^{[l]}\left(f_{1}\right)=$ $\Pi_{G}^{[l]}\left(f_{2}\right)$.

Proof. In view of the naturality of the construction, this amounts to prove that $\Pi_{G}^{[l]}(h)=$ id for the map $h:(X, A) \times I \rightarrow(X, A) \times I$ given by $(x, t) \mapsto(x, 0)$. Let $(E, F, l, c)$ be a cocycle representing an element in $\Pi_{G}^{[l]}(X, A \times I)$. In the notation of proposition 2.15, there exist unitary morphisms $u: E \rightarrow h^{*}(E), v: F \rightarrow h^{*}(F)$ covering the identity $X \times I \rightarrow X \times I$ such that the restrictions to $E_{0}, F_{0}$ are the respective identities. Note that the composition $f=h^{*}(E) \stackrel{u^{-1}}{\rightarrow} E \stackrel{l+c}{\rightarrow} F \stackrel{v^{-1}}{\rightarrow} h^{*}(F)$ is homotopic to $h^{*}(l+c)$ relative to $h^{*}(l)$. After checking out the conditions for the definition of $\Pi_{G}^{[l]}(X)$, one has that the equivalence classes $\Pi_{G}^{[l]}(h)(l+c)=$ $[f]=[l+c]$ agree.

Definition 5.6. Let $l: E \rightarrow F$ be a fixed representative of a class in $K O(X)_{0}^{G}$. The group $\Pi_{G}^{[l+1]}(X)$ is defined to be the group of equivalence classes represented 
by cocycles defined over $X$, which admit a representation by a cocycle $(E \oplus$ $\left.\mathbb{R}, E, l \circ \operatorname{proj}_{E}, c\right)$, where $\operatorname{proj}_{E}$ denotes the projection onto the subspace $E$.

We construct a suspension isomorphism

$$
\sigma_{[l]}^{X, A}: \Pi_{G}^{[l]}(X, A) \rightarrow \Pi_{G}^{[l]+1}((X, A) \times(I,\{0,1\}))
$$

Given $l+c: E \rightarrow F \in \Pi_{G}^{[l]}\left((X, A)\right.$, form the bundle $E^{\prime}=E \oplus \mathbb{R}$, denote by $p$ the projection $E \oplus \mathbb{R} \rightarrow E$ and define the map $\sigma_{[l]}(l+c): E^{\prime} \times I \rightarrow E^{\prime} \times I$ defined as $(e, t) \mapsto p \circ l+(\log (t)-(\log (-t)))(c(p(v))$. By this means, we obtain a cocycle $(E \oplus$ $\mathbb{R}, E, p \circ l, C)$ which extends to the fibrewise one-point compactifications, beeing trivial on the required subspace. Given an element $l+c \in \Pi_{G}^{[l+1]}((X, A) \times I,\{0,1\})$, consider a unitary, fibrewise linear cocycle $u: E \rightarrow E_{0} \times I v: F \rightarrow F_{0}$ covering the identity $X \times I \rightarrow X \times I$, which restricted over the subspace $A \times I \cup X \times\{0,1\}$ is the identity map. The map constructed as $E_{0} \times I \stackrel{u^{-1}}{\rightarrow} E \stackrel{\varphi+c}{\rightarrow} F \stackrel{v}{\rightarrow} F_{0} \times I$ determines an inverse for $\sigma_{[l]}^{(X, A)}$.

A coboundary map is defined as the composition

$$
\begin{gathered}
\Pi_{G}^{[l]}(A) \stackrel{\sigma_{G}^{n}(A)}{\longrightarrow} \Pi_{G}^{[l+1]}(A \times I, A \times\{0,1\}) \\
\stackrel{\Pi_{G}^{[l+1]}\left(i_{1}\right)^{-1}}{\longrightarrow} \Pi_{G}^{[l+1]}\left(X \cup_{A \times\{0\}} A \times I, X \coprod A \times\{1\}\right) \\
\stackrel{\Pi_{G}^{[l+1]}\left(i_{2}\right)}{\longrightarrow} \Pi_{G}^{[l+1]}\left(X \cup_{A \times\{0\}} A \times I, A \times\{1\}\right) \\
\stackrel{\Pi_{G}^{[l+1]}\left(\operatorname{pr}_{1}\right)^{-1} \Pi_{G}^{[l+1]}(X, A)}{\longrightarrow}
\end{gathered}
$$

Where the maps, $i_{n}$ for $n=0,1$ are the inclusions, and $p r_{1}$ is the projection . The maps $\Pi_{G}^{n+1}\left(i_{n}\right)$ are bijective by excision and $\Pi_{G}^{n+1}\left(\mathrm{pr}_{1}\right)$ is bijective because of homotopy invariance.

We analize now induction structures. In order to define the induction structure in equivariant cohomotopy, we restrict ourselves to the case where $G$ is a Lie group.

Let $\alpha: H \rightarrow G$ be a group homomorphism and $X$ be an $H$-CW complex. The induced space $\operatorname{ind}_{\alpha} X$, is the $G$-CW complex defined as the quotient space obtained from $G \times X$ by the right $H$-action given by $(g, x) \cdot h=\left(g \alpha(h), h^{-1} x\right)$. 
Nonlinearity, Proper Actions and Equivariant Stable Cohomotopy 405

Proposition 5.7. Let $\alpha: H \rightarrow G$ be a proper Lie group homomorphism. Then there exists a group homomorphisn

$$
\Pi_{G}^{[l]}\left(\operatorname{ind}_{\alpha}(X, A)\right) \rightarrow \Pi_{H}^{[l]}(X, A)
$$

which satisfies

(i) Bijectivity. If $\operatorname{ker}(\alpha)$ acts freely on $(X, A)$, then the map is an isomorphism.

(ii) Compatibility with the boundary homomorphisms. $\delta_{H}^{[l]} \circ \operatorname{Ind}_{\alpha}=\operatorname{ind}_{\alpha} \circ \delta_{G}^{[l]}$.

(iii) Functoriality. If $\beta: G \rightarrow K$ is a group homomorphism, then the diagram commutes:

(iv) Compatibility with conjugation.For any $g \in G$, the homomorphism

$$
\left.\operatorname{ind}_{\left.c_{(g)}\right): G \rightarrow G} \Pi_{G}^{[l]}(X, A) \rightarrow \Pi_{G}^{[l]}(\text { ind })_{\mathrm{c}(\mathrm{g}): \mathrm{G} \rightarrow \mathrm{G}}(\mathrm{X}, \mathrm{A})\right)
$$

agrees with the map $\Pi_{G}^{[l]}\left(f_{2}\right)$, where $f_{2}:(X, A) \rightarrow \operatorname{ind}_{c(g): G \rightarrow G}$ sends $x$ to $\left(1, g^{-1} x\right)$ and $c(g)$ is the conjugation isomorphism in $G$ associated to $g$

Proof. (i) The map i $: X \rightarrow \operatorname{ind}_{\alpha}(X)\left(x \mapsto\left(1_{G}, X\right)\right.$ induces a group homomorphism $\Pi_{G}^{*}\left(\operatorname{ind}_{\alpha} X, A\right) \rightarrow \Pi_{H}^{[l]}(X, A)$. An inverse is given by the map which associates to a linear cocycle $(E, F, \varphi)$ the cocycle $(E / H, F / H, \varphi / H)$. It is easy to show that this is the case for the perturbation and that this still satisfies the boundedness condition. We point out that we state this fact for proper actions of Lie groups, see remark below.

(ii) Follows from the naturality of the induced bundle construcctions.

(iii) Follows from the functoriality of the induced vector bundle construction.

(iv) Compatibility with conjugation. Follows from element chasing in the diagram

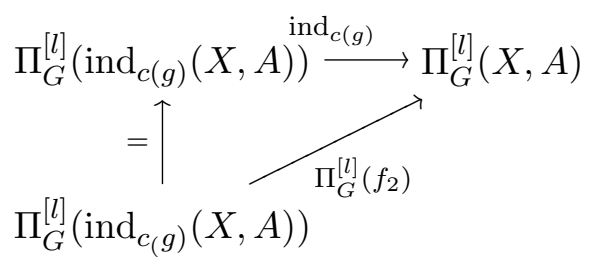

where $f_{2}:(X, A) \rightarrow \operatorname{ind}_{c(g)}(X, A)$ is given by $x \mapsto\left(1, g^{-1} x\right)$ and $c(g)\left(g^{-1}\right)=$ $g g^{\prime} g^{-1}$. 
Remark 5.8. N.C. Phillips proves in [22], corollary 8.5 , p. 131 a more general result for equivariant $K$-theory, allowing proper actions of locally compact groups as input, instead of only Lie groups. The adaptation of these methods is certainly plausible. The main problematic point is the induction structure. We cannot guarantee the local triviality of bundles $E / H \rightarrow X / H$ unless $H$ is Lie, see [21].

\section{The PARAMETRIZED SchWARZ-INDEX}

Several approaches have been proposed towards the definition of equivariant cohomotopy theory for proper actions. Lück [14] uses finite dimensional bundles. This allows to deal with the difficulties appearing in the case where a discrete group acts on a finite $G$-CW complex. We briefly recal this approach

Fix an equivariant, proper $G$-CW complex. Form the category

$\operatorname{SPHB}^{G}(X)$ which has as as objects $G$ - equivariant, finite dimensional real vector bundles over $X$. Denote by $S^{\xi}$ the one point compactification bundle asociated to the vector bundle $\xi$. A morphism from $\xi: E \rightarrow X$ to $\mu: F \rightarrow X$ is a bundle map $S^{\xi} \rightarrow S^{\mu}$ covering the identity in $X$, which preserves fiberwise the basic points. A homotopy between the morphisms $u_{0}, u_{1}$ is a $G$-bundle map $h: S^{\xi} \times[0,1] \rightarrow S^{\mu}$ from the bundle $S^{\xi} \times[0,1] \rightarrow[0,1] \times X$ to the bundle $S^{\mu}$ covering the projection $X \times[0,1] \longrightarrow X$ and preserving the base points on every fiber such that its restriction to $X \times\{i\}$ is $u_{i}$ for $i=0,1$. Let $\underline{\mathbb{R}^{n}}$ be the trivial vector bundle over $X$, which is furnished with the trivial action of $G$. Two morphisms of the form

$$
S^{\xi_{i} \oplus \underline{\mathbb{R}^{k_{i}}}} \rightarrow S^{\xi_{i} \oplus \underline{\mathbb{R}^{k_{i}+n}}}
$$

are said to be equivalent if there are objects $\mu_{i}$ in $\operatorname{SPHB}^{G}(X)$ and an isomorphism of vector bundles $\nu: \mu_{0} \oplus \xi_{0} \cong \mu_{1} \oplus \xi_{1}$ such that the following diagram of 
morphisms in $\operatorname{SPHB}^{G}(X)$ commutes up to homotopy

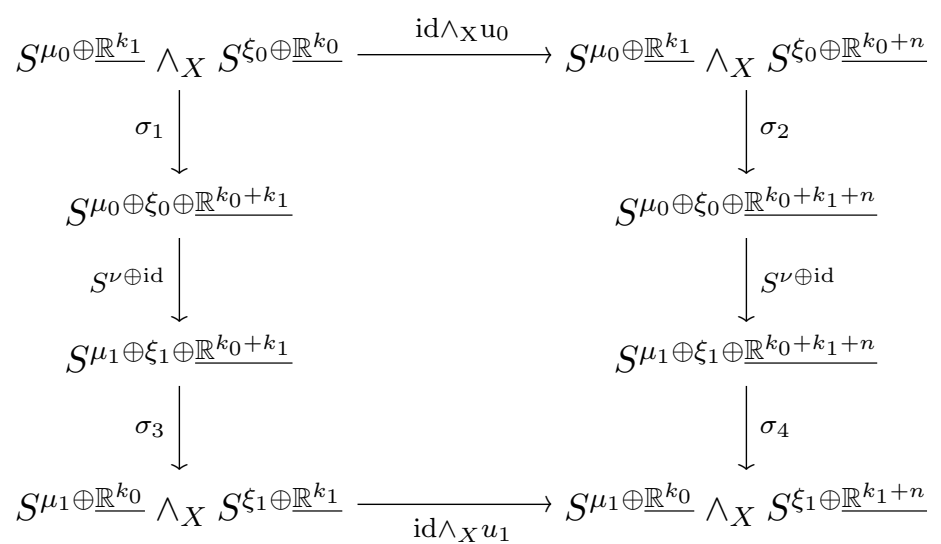

where the isomorphisms $\sigma_{i}$ are determined by the fiberwise defined homeomorphism $S^{V \oplus W} \approx S^{V} \wedge S^{W}$ and the associativity of smash products, which holds for every pair of representations $V, W$. We recall now W. Lück's definition of equivariant cohomotopy:

Definition 6.1. Let $X$ be a $G$-CW complex, where $G$ is a discrete group and $X$ is finite. We define its $n$-th G-equivariant stable cohomotopy group $\pi_{G}^{n}(X)$ as the set of homotopy clases of equivalence classes of morphisms $u: S^{\xi \oplus \underline{\mathbb{R}^{k}}} \rightarrow$ $S^{\xi \oplus \underline{\mathbb{R}^{k+n}}}$ under the above mentioned relation. For a $G$-CW pair, $(X, A)$ we define $\Pi_{G}^{n}(X, A)$ as the equivalence classes of morphisms which are trivial over $A$, i.e.

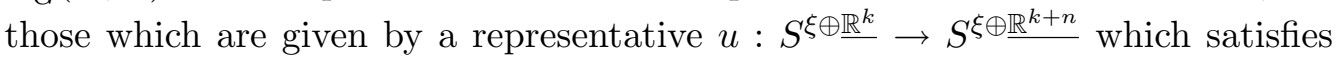
that over every point $a \in A$, the map $u_{a}: S^{\xi_{a} \oplus \mathbb{R}^{k}} \rightarrow S^{\xi_{a} \oplus \underline{\mathbb{R}^{k+n}}}$ is constant

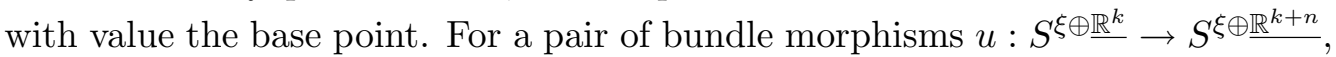

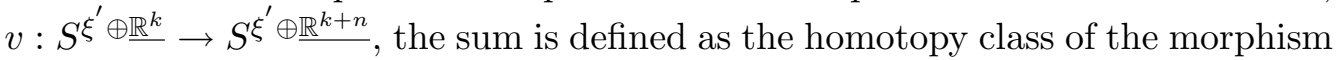

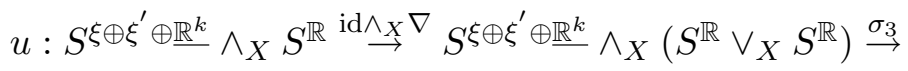

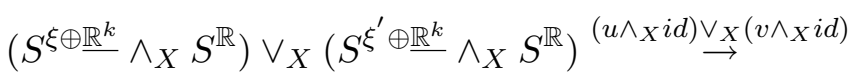

$$
\begin{aligned}
& S^{\xi \oplus \xi^{\prime} \oplus \underline{\mathbb{R}^{k+n}}} \wedge_{X} S^{\mathbb{R}}
\end{aligned}
$$

where $\sigma_{3}$ is the canonical isomorphism given by the fiberwise distributivity and associativity isomorphisms and $\nabla$ denotes the pinching map $S^{\mathbb{R}} \rightarrow S^{\mathbb{R}} \vee S^{\mathbb{R}}$. The relative version for elements lying in the group of a pair, $\pi_{G}^{n}(X, A)$ translates word by word when one sets all sphere bundles and morphisms to be trivial over $A$. 
Our approach extends the notions proposed first by Lück and solves its principal problem, namely: the the lack of finite dimensional $G$-vector bundles to represent excisive $G$-cohomology theories. The crucial result in this is the construction of an index theory in the context of parametrized nonlinear analysis. Previous versions of this index theory (restricted to the unparametrized and $S^{1}$ equivariant case) were constructed in [3], [26], [19] from where we adopt the crucial ideas.

We prepare its definition with the following facts, which are parametrized versions of the discussion in page 5 and Lemma 2.5 in [3]. We also use the notation from that article. In this section, all groups are discrete and all $G$-CW complexes are finite.

Definition 6.2. Let $l: E \rightarrow E$ be a Fredholm morphism over a proper $G$-CW complex $X$, equipped with some invariant riemannian metric. For any finite dimensional $G$-vector subbundle $\xi$ embedded as direct summand, the orthogonal spherical retraction associated to $\xi, \rho_{\xi}: S^{E}-S^{\xi^{\perp}} \rightarrow S^{\xi}$ is defined to be the map given on every fiber $x$ as $w \mapsto|w| \operatorname{Proj}_{\xi}(w)$

The following is a parametrized version of lemma 2.5 in [3], compare also Corollary 3.11 in [19]:

Proposition 6.3. Let $G$ be a discrete group acting on a finite $G$-Cw complex. Let $f=l+c: E \rightarrow E$ be a fibrewise compact perturbation of the linear Fredholm morphism $l$ defined in a $G$-Hilbert bundle $E$ over the proper $G$-space $X$. Then, there exist finite dimensional $G$-vector bundles $\xi \subset E$ such that

- $\xi$ spans together with the image of $l$ the G-Hilbert bundle E.

- Given a fibrewise inclusion as orthogonal summand in a finite dimensional vector bundle $\xi \rightarrow \tau, \xi \oplus \zeta=\tau$, the restricted map $f: S^{l^{-1}(\xi)} \rightarrow S^{\xi}$ sends the unit sphere $S^{\xi^{\perp}}$ to the orthogonal complement of $\xi$.

- The maps $\left.\rho_{\tau} f\right|_{l^{-1}(\tau)}$ and $i d_{\zeta} \wedge_{X} \rho_{\xi}$ are G-homotopic over $X$.

Proof. $\quad$ (i) Denote by $D$ the unit ball bundle in $E$. Due to the boundedness condition, the map $f^{-1}\left(D_{x}\right)$ is bounded over any point $x \in X$. Hence, the closure $C_{x}$ of its image under the compact map $c$ is fibrewise compact. It follows that given $\epsilon<\frac{1}{4}$ there exist continuous sections $\left\{\phi_{i}: X \rightarrow E\right\}_{i=1}^{n}$ such that $C_{x} \subset B_{\epsilon}\left(\phi_{i}(x)\right)$. One can furthermore assume that $\left\{\phi_{i}(x)\right\}$ 
Nonlinearity, Proper Actions and Equivariant Stable Cohomotopy 409

form a linearly independent set and the linear subspace spanned by them intersects trivially the orthogonal complement of the image, $l(E)^{\perp}$ on each fiber. The finite dimensional bundle associated to the fredholm morphism given as the projection onto $l(E)_{x}^{\perp} \oplus\left\langle\phi_{1}, \ldots, \phi_{n}(x)\right\rangle$ satisfies the required properties.

(ii) If $w_{x} \in S_{x}^{\tau^{\perp}}$ is in the image of $\left.f\right|_{S^{l^{-1}(\tau)}}$, then $\left.f^{-1}\left(w_{x}\right)\right) \cap l^{-1}(\tau)$ will be mapped under $\left.f\right|_{l^{-1}}$ to a subspace of $\tau_{x}+\left\langle\left\{\phi_{i}\right\}\right\rangle$. So, $w_{x}$ will be contained in $S^{\tau^{\perp}} \cap \tau_{x}+\left\langle\left\{\phi_{i}\right\}\right\rangle$, which is not possible, because the distance between these subspaces is greater than $1-\epsilon>\frac{3}{4}$.

(iii) In view of the slice theorem and the local triviality of the $G$-Hilbert bundles involved, we can cover the space $X$ with invariant neighborhoods for which there is a map $U_{x} \rightarrow G / H$, and the bundle over $U_{x}$ is the

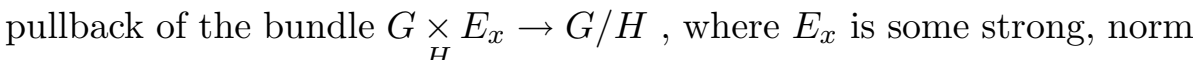
continuous representation of $H$ in a Hilbert space. Hence, we can restrict ourselves to bundles over an orbit. In the notation of the previous part, there is a retraction $\rho_{\tau_{x}}: S^{\tau_{x}} \rightarrow S^{E_{x}}-S^{\tau_{x}^{\perp}}$. We consider the isomorphism $l^{-1}\left(\tau_{x}\right) \cong \zeta_{x} \oplus l^{-1}\left(\xi_{x}\right)$ given by $w_{x}^{\prime} \mapsto\left(l \circ\left(1-\operatorname{pr}_{l^{-1}\left(\xi_{x}\right)} w_{x}^{\prime}, \operatorname{pr}_{l^{-1}\left(\xi_{x}\right)} w_{x}^{\prime}\right)\right.$. We claim that after this isomorphism, the maps $\operatorname{id}_{\zeta_{x}} \rho_{\xi_{x}}\left(\left.f\right|_{l^{-1}\left(\xi_{x}\right)}\right)$ and $\left.f\right|_{S^{-1}\left(\tau_{x}\right)}$ are homotopic. Consider for this a ball $D \subset E$ which contains the inverse image $f^{-1}\left(D_{1}(0)\right)$ of the unitary ball We define the homotopy $h: D \times I \rightarrow S^{E}-S^{\tau^{\perp}}$ as follows

$$
h\left(w_{x}, t\right)= \begin{cases}l+\left[(1-3 t) \mathrm{id}_{E_{x}}+(3 t) \mathrm{pr}_{\xi_{x}}\right] \circ c & t \in\left[0, \frac{1}{3}\right] \\ l+\operatorname{pr}_{\xi_{x}} \circ c\left[(2-3 t) \operatorname{id}_{l-1} \xi_{x}+(3 t-1) \operatorname{pr}_{l^{-1}\left(\xi_{x}\right)}\right] & t \in\left[\frac{1}{3}, \frac{2}{3}\right] \\ \operatorname{pr}_{\zeta_{x}} \circ l+\left[(3-3 t) \mathrm{pr}_{\xi_{x}}+(3 t-2) \rho_{\zeta_{x}} \circ(l+c) \circ \mathrm{pr}_{l-1}\left(\zeta_{x}\right)\right. & t \in\left[\frac{2}{3}, 1\right]\end{cases}
$$

Since $S_{x}^{E}-D \cap \tau_{x}^{\perp}$ is contractible, the homotopy above can be extended to a homotopy $S^{l^{-1}(\tau)} \times I \rightarrow S^{E}-S^{\tau^{\perp}} \simeq S^{\tau}$, as needed.

Using appropiate rescaling homotopies we have:

Proposition 6.4. The homotopy type of an orthogonal spherical retraction does not depend on the choice of a riemannian metric.

Definition 6.5 (Parametrized Schwarz index). Let $G$ be a discrete group acting on the proper, finite $G$-CW complex $X$. Denote by $(E, F, l, k)$ a non-linear cocycle for the equivariant cohomotopy theory over the proper, finite $G$-CW complex $X$, 
where $l$ is a linear morphism whose index bundle is trivial of virtual dimension p. Let $\xi$ be a finite dimensional $G$-vector bundle as constructed in proposition 6.3. The parametrized Schwartz index is the class of the element.

$$
\left[\left.p_{\xi}(l+k)\right|_{l^{-1}(\xi)}\right]: S^{l^{-1}(\xi)} \rightarrow S^{\xi} \in \pi_{G}^{p}(X)
$$

in the equivariant cohomotopy group as, introduced by Lück in [14]. This construction does not depend of the choice of the finite dimensional vector bundle as a consequence of part 3 of proposition 6.3 .

We are now able to state our main result:

Theorem 6.6. Let $G$ be a discrete group acting on a $G$-CW pair $(X, A)$. Denote by $[l]$ the $\mathrm{KO}_{G}$-theoretical class of a Fredholm morphism whose fibrewise index is a trivial virtual vector bundle of dimension $p$. The parametrized Schwartz index gives an isomorphism

$$
\Pi_{G}^{[l]}(X, A) \rightarrow \pi_{G}^{p}(X, A)
$$

Proof. We construct a natural inverse map. Using proposition 2.12, we can add finite dimensional vector bundles and assume that the morphism $u: S^{\xi \oplus \mathbb{R}^{n+p}} \rightarrow$ $S^{\xi \oplus \mathbb{R}^{n}}$ is homotopic to a map such for which on every fiber, the only preimage of the basis point $\infty$ is $\infty$. We denote by $c: \Gamma\left(\xi \oplus \mathbb{R}^{n+p}\right) \rightarrow \Gamma\left(\xi \oplus \mathbb{R}^{n}\right)$ the (possibly nonlinear) map obtained by restricting $u$ fibrewise to the complement of the point at infinity.

Consider the projection operator $P_{\xi \oplus \mathbb{R}^{n}}: \Gamma\left(\xi \oplus \mathbb{R}^{n+p}\right) \rightarrow \Gamma\left(\xi \oplus \mathbb{R}^{n}\right)$. Let $\mathcal{H}$ be the stable $G$-Hilbert space of proposition 2.12. Recall that $\Gamma(\eta) \oplus \Gamma(X \times \mathcal{H}) \cong$ $\Gamma(X \times \mathcal{H})$ for all $G$-Hilbert bundles $\eta$, due to the proper stabilization theorem. The map of $C(X)$ - $G$ - Hilbert modules

$P_{\xi \oplus \mathbb{R}^{n}} \otimes_{C(X)}$ id $: \Gamma\left(\xi \oplus \mathbb{R}^{n+p}\right) \otimes \Gamma(X \times \mathcal{H}) \rightarrow \Gamma\left(\xi \oplus \mathbb{R}^{n}\right) \otimes \Gamma(\mathcal{H} \times X) \cong \Gamma(X \times \mathcal{H})$ determines up to precomposition with the proper stability isomorphism a Fredholm morphism $l: X \times \mathcal{H} \rightarrow X \times \mathcal{H}$, which represents a class for which the index bundle is trivial of virtual dimension $p$. We denote by $C$ the proper, compact, nonlinear map defined on every fiber by

$$
\Gamma(X \times \mathcal{H}) \stackrel{\cong}{\rightrightarrows} \Gamma(X \times \mathcal{H}) \oplus \Gamma\left(\xi \oplus \mathbb{R}^{n+p}\right) \stackrel{0 \oplus c}{\rightarrow} \Gamma(X \times \mathcal{H}) \oplus \Gamma\left(\xi \oplus \mathbb{R}^{n}\right) \rightarrow \Gamma(X \times \mathcal{H})
$$

where the first map is given by the proper stabilization isomorphism, and the second one is the inclusion in the first factor of the direct sum followed by the 
proper stabilization isomorphism. The cocycle associated to $u$ is $(X \times \mathcal{H}, X \times$ $\mathcal{H}, l, C)$.

Remark 6.7. In the case of compact Lie groups, one may assume that the index bundle ker $-\operatorname{coker}(l)$ has the form of a trivial bundle $X \times V$, where $V$ is a $G$ - representation. The equivariant Schwartz index identifies this with the usual definition for equivariant cohomotopy groups for finite $G$-complexes. Specializing to the trivial group and the one-point space, this theorem can be traced to the main result in [25], and in [3] one finds an $S^{1}$-equivariant version which basically deals with all problems on compact Lie groups. In view of this correspondence, we use in the rest of this note the notation $\Pi$ for cohomotopy or its equivariant generalizations.

\section{A Burnside Ring for Lie Groups}

We now define a Burnside ring in operator theoretical terms for non compact Lie groups. We first recall the definition for compact Lie groups, which was first introduced by Tom Dieck in [27].

Definition 7.1. Let $G$ be a compact Lie group. Consider the folowing equivalence relation on the collection of finite $G-C W$ complexes. $X \sim \mathrm{Y}$ if and only if for all $H \subset G$, the spaces $X^{H}$ and $X^{H}$ have the same Euler characteristic. Let $A(G)$ be the set of equivalence classes. Disjoint union and cartesian product of complexes are compatible with this equivalence relation and induce composition laws on $A(G)$. It is easy to verify that $A(G)$ together with these composition laws is a commutative ring with identity. The zero element is represented by a complex $X$ such that the Euler characteristic $\chi\left(X^{H}\right)$ is zero for each $H \subset G$. If $K$ is a space with trivial $G$-action and $\chi(K)=-1$, then $X \times K$ represents the additive inverse of $X$ in $A(G)$.

We collect some information about the algebraic structure of the Burnside ring in the followiing results, which have been published by Tom Dieck in [29], pages 240250 and 256, respectively.

Proposition 7.2. $\quad$ (i) As abelian group $A(G)$ is the free abelian group on $G / H$, where $H \in \Phi(G)$ and $\Phi(G)$ denotes the space of conjugacy classes 
of subgroups such that $N(H, G) / H$ is finite, where $N(H, G)$ denotes the normalizer of $H$ in $G$.

(ii) There is a character map $\operatorname{char}_{G}: A(G) \rightarrow \operatorname{Map}(\Phi(G), \mathbb{Z})$, where $\Phi(G)$, the space of closed subgroups of $G$ carries the Hausdorff metric (in particular it is a compact Hausdorff space). And $\operatorname{char}_{G}(X)$ is defined by $H \mapsto X^{H}$.

Proposition 7.3. By means of the character map, the elements of the Burnside ring can be identified with sums

$$
\sum_{K} n(H, K) \chi\left(X^{K}\right) \cong 0 \bmod |N H / H|
$$

where the sum is over conjugacy classes $(K)$ such that $H$ is normal in $K$, $K / H \subset N_{H, G} / H$ is cyclic, the integer numbers $n(H, K)$ are defined to be

$$
n(h, K)=|\operatorname{Gen}(K / H)|\left|W_{H, G} / N_{W_{K / H}, W_{H, G}}\right|
$$

and $\operatorname{Gen}(Z)$ denotes the cardinality of the generators of the finite cyclic group $Z$. In particular, the rationalized Burnside ring $A(G) \otimes \mathbb{Q}$ can be identified with the ring of continuous rational functions defined on $\Phi(G)$

Theorem 7.4 ( [28], p.188). Let $G$ be a compact Lie group. There is an isomorphism

$$
\Pi_{G}^{0}(\{*\}) \rightarrow A(G)
$$

Remark 7.5 (The spectral sequence). Let $X$ be a proper $G$-CW complex. There is an equivariant Atiyah-Hirzebruch spectral sequence which converges to $\Pi_{G}^{n}(X)$ and whose $E^{2}$-term is given in terms of Bredon cohomology

$$
E_{2}^{p, q}=H_{\mathbb{Z} O r G}^{p}\left(X, \Pi_{?}^{q}\right)
$$

AppLied to the universal proper $G$-space $\underline{\mathrm{E}} G$ :

$$
E_{2}^{p, q}=H_{\mathbb{Z} \mathcal{S U} \mathcal{B}_{\mathcal{C O M}(G)}^{p}}\left(\underline{\mathrm{E}} G, \Pi_{?}^{q}\right)
$$

where $\Pi_{?}^{0}$ is the contravariant coefficient system $H \mapsto \pi_{H}^{0}$.

There is a canonical identification

$$
H_{\mathbb{Z} \mathcal{S U} \mathcal{B}_{\mathcal{C O M}(G)}}\left(\underline{\mathrm{E}} G, \Pi_{?}^{0}\right) \cong \lim _{H \in \mathcal{C O} \mathcal{M}} \Pi_{H}^{0}
$$


The edge homomorphism of the spectral sequence defines a map edge ${ }^{G}$ : $\Pi_{G}^{0}(\underline{\mathrm{E} G}) \rightarrow A_{\text {inv }}(G)$. Several known results of the spectral sequence go trough. Among them, as in case of discrete groups, the edge homomorphism is a rational isomorphism.

Definition 7.6 (An operator theoretical Burnside Ring). Let $G$ be a locally compact group. The operator theoretical burnside ring of $G, A^{\mathrm{op}}(G)$ is the 0 dimensional equivariant cohomotopy theory of the classifying space of proper actions $\underline{E} G$. In symbols

$$
A^{\mathrm{op}}(G)=\Pi_{G}^{0}(\underline{\mathrm{E}} G)
$$

The augmentation ideal $\hat{\mathbf{I}}_{G} \subset \Pi_{G}^{0}(\underline{\mathrm{E}} G)$ is defined to be the kernel of the composition of the restriction to the oth- skeleton of the classifying space and the restriction to the trivial group

$$
\Pi_{G}^{0}(\underline{\mathrm{E}} G) \rightarrow \Pi_{G}^{0}\left(\underline{\mathrm{E}} G_{0}\right) \rightarrow \Pi_{\{e\}}^{0}\left(\underline{\mathrm{E}} G_{0}\right)
$$

Example 7.7 (The group $S l_{2}(\mathbb{R})$ ). Recall that the group $S l_{2}(\mathbb{R})$ is defined to be the group of real $2 \times 2$-matrices with determinant 1 . It is a Lie group of dimension 3 and has one connected component. The maximal compact subgroup is $\mathrm{S}^{1}=\mathrm{SO}_{2}$.

As $S l_{2}(\mathbb{R})$ is almost connected, a model for $\mathrm{E}_{\mathrm{COM}_{\mathcal{M}} \mathrm{Sl}_{2}}$ is $S l_{2}(\mathbb{R}) / S O_{2} \approx \mathbb{R}^{2}$, which can be handled as the upper-half plane model for the 2-dimensional hyperbolic space. Note that this is a zero-dimensional proper $C W$-complex. From the equivariant Atiyah- Hirzebruch Spectral Sequence follows that the edge homomorphism

$$
\operatorname{edge}^{S l_{2}(\mathbb{R})}: \Pi_{S l_{2}(\mathbb{R})}^{0}\left(\underline{E} G S l_{2}(\mathbb{R})\right) \rightarrow \lim _{\operatorname{inv} H \in \mathcal{C O M}\left(S l_{2}(\mathbb{R})\right)} \Pi_{H}(p t)
$$

is an isomorphism. On the other hand, since $S^{1}$ is a final object in the category of compact subgroups of $S l_{2}(\mathbb{R})$, we have

$$
\begin{gathered}
A^{\mathrm{op}}\left(S l_{2}(\mathbb{R})\right) \cong A\left(S^{1}\right) \\
A\left(S^{1}\right) \cong \mathbb{Z}
\end{gathered}
$$

is a well known fact. 


\section{Extending one version of the Segal conjecture for lie groups}

The Segal conjecture for finite groups, proven in 1984 [5] states the existence of an isomorphism between a certan completion of the Burnside ring and the 0thstable cohomotopy of the classifying space:

Theorem 8.1 (Carlsson, 1984). The Segal conjecture is true for finite groups. That is, there is an isomorphism

$$
A(G)_{\hat{I}_{G}} \cong \Pi_{\{1\}}^{0}(\mathrm{~B} G)
$$

In the case of Lie groups, this conjecture is known to be false in this generality [9]. However, a weaker version was obtained by Feshbach in [9] and later refined by Bauer in [2]. The statement is :

Theorem 8.2. [Segal conjecture for compact Lie groups]

Let $G$ be a compact Lie group with maximal torus $T$ of dimension $n$ and Weyl group $W=N_{T, G} / T$. Let $\rho: W \rightarrow G l_{n}(\mathbb{Z})$ be a representation which gives rise to the action of $W$ on $T \approx \mathbb{R}^{n} / \mathbb{Z}^{n}$. Suppose that $\rho$ does not originate at a generalized quaternion group of order $2^{n}$. Then the map

$$
A(G)_{\hat{\mathbf{I}}_{G}} \rightarrow \Pi_{\{1\}}^{0}(\mathrm{BG})
$$

has dense image in the skeletal filtration.

We extend this theorem in the following direction:

Theorem 8.3. [Segal Conjecture for almost connected Lie groups] The Segal conjecture is true for (non compact) Lie groups with finitely many components. That is, there is a map

$$
A^{\mathrm{op}}(G)_{\hat{\mathbf{I}}_{G}} \rightarrow \Pi_{\{1\}}^{0}(\mathrm{BG})
$$

with dense image in the skeletal filtration whenever a maximal compact subgroup of $G$ satisfies the hypotheses of theorem 8.2.

Proof. Let $G$ be a Lie group with finitely many components. Then

(i) There is up to conjugacy a unique maximal compact subgroup $K$ of $G$. Any other compact subgroup is subconjugated to $K$.

(ii) There exist diffeomorphisms $G \approx G / K \times K$ and $G / K \approx \mathbb{R}^{k}$. 
See [11], Theorem 3.1 p 180 for a proof of this. Hence, the space $G / K$ carries a proper action, and in particular, the induction isomorphism gives an isomorphism $A^{o p}(G) \cong A(K)$, where $A(K)$ stands for the Burnside ring in the sense of Tom Dieck [27]. On the other hand, the classifying spaces BG and BK have the same homotopy type. Hence the map

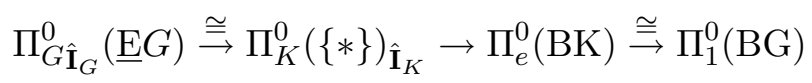

has dense image as a consequence of theorem 8.2.

\section{REFERENCES}

[1] M. Atiyah and G. Segal. Twisted K-theory. Ukr. Mat. Visn., 1(3):287-330, 2004.

[2] S. Bauer. On the Segal conjecture for compact Lie groups. J. Reine Angew. Math., 400:134$145,1989$.

[3] S. Bauer and M. Furuta. A stable cohomotopy refinement of Seiberg-Witten invariants. I. Invent. Math., 155(1):1-19, 2004.

[4] M. S. Berger. Nonlinearity and functional analysis. Academic Press [Harcourt Brace Jovanovich Publishers], New York, 1977. Lectures on nonlinear problems in mathematical analysis, Pure and Applied Mathematics.

[5] G. Carlsson. Equivariant stable homotopy and Segal's Burnside ring conjecture. Ann. of Math. (2), 120(2):189-224, 1984.

[6] K. Deimling. Nonlinear functional analysis. Springer-Verlag, Berlin, 1985.

[7] A. Dold. The fixed point index of fibre-preserving maps. Invent. Math., 25:281-297, 1974.

[8] H. Emerson and R. Meyer. Equivariant representable K-theory. J. Topol., 2(1):123-156, 2009.

[9] M. Feshbach. The Segal conjecture for compact Lie groups. Topology, 26(1):1-20, 1987.

[10] M. Furuta. Finite dimensional approximations in geometry. In Proceedings of the International Congress of Mathematicians, Vol. II (Beijing, 2002), pages 395-403, Beijing, 2002. Higher Ed. Press.

[11] G. Hochschild. The structure of Lie groups. Holden-Day Inc., San Francisco, 1965.

[12] J. Ize. Equivariant degree. In Handbook of topological fixed point theory, pages 301-337. Springer, Dordrecht, 2005.

[13] W. Lück. Transformation groups and algebraic K-theory, volume 1408 of Lecture Notes in Mathematics. Springer-Verlag, Berlin, 1989. Mathematica Gottingensis.

[14] W. Lück. The Burnside ring and equivariant stable cohomotopy for infinite groups. Pure Appl. Math. Q., 1(3):479-541, 2005.

[15] W. Lück. Survey on classifying spaces for families of subgroups. In Infinite groups: geometric, combinatorial and dynamical aspects, volume 248 of Progr. Math., pages 269-322. Birkhäuser, Basel, 2005. 
[16] A. Malcev. On isomorphic matrix representations of infinite groups. Rec. Math. [Mat. Sbornik] N.S., 8 (50):405-422, 1940.

[17] W. Marzantowicz. On the nonlinear elliptic equations with symmetry. J. Math. Anal. Appl., 81(1):156-181, 1981.

[18] J. P. May. Equivariant homotopy and cohomology theory, volume 91 of CBMS Regional Conference Series in Mathematics. Published for the Conference Board of the Mathematical Sciences, Washington, DC, 1996. With contributions by M. Cole, G. Comezaña, S. Costenoble, A. D. Elmendorf, J. P. C. Greenlees, L. G. Lewis, Jr., R. J. Piacenza, G. Triantafillou, and S. Waner.

[19] C. Okonek and A. Teleman. Cohomotopy invariants and the universal cohomotopy invariant jump formula. J. Math. Sci. Univ. Tokyo, 15(3):325-409, 2008.

[20] A. Y. Ol'shanskiı. Groups of bounded period with subgroups of prime order. Algebra $i$ Logika, 21(5):553-618, 1982.

[21] R. S. Palais. On the existence of slices for actions of non-compact Lie groups. Ann. of Math. (2), 73:295-323, 1961.

[22] N. C. Phillips. Equivariant K-theory for proper actions, volume 178 of Pitman Research Notes in Mathematics Series. Longman Scientific \& Technical, Harlow, 1989.

[23] C. Prieto. $K \mathrm{O}(B)$-graded stable cohomotopy over $B$ and $R \mathrm{O}(G)$-graded $G$-equivariant stable cohomotopy: a fixed point theoretical approach to the Segal conjecture. In The Lefschetz centennial conference, Part II (Mexico City, 1984), volume 58 of Contemp. Math., pages 89-108. Amer. Math. Soc., Providence, RI, 1987.

[24] H. Schröder. K-theory for real $C^{*}$-algebras and applications, volume 290 of Pitman Research Notes in Mathematics Series. Longman Scientific \& Technical, Harlow, 1993.

[25] A. S. Švarc. On the homotopic topology of Banach spaces. Dokl. Akad. Nauk SSSR, 154:6163, 1964.

[26] M. Szymik. Characteristic cohomotopy classes for families of 4-manifolds. Forum Math., 22(3):509-523, 2010.

[27] T. tom Dieck. The Burnside ring of a compact Lie group. I. Math. Ann., 215:235-250, 1975.

[28] T. tom Dieck. Transformation groups and representation theory, volume 766 of Lecture Notes in Mathematics. Springer, Berlin, 1979.

[29] T. tom Dieck. Transformation groups, volume 8 of de Gruyter Studies in Mathematics. Walter de Gruyter \& Co., Berlin, 1987.

[30] H. Ulrich. Fixed point theory of parametrized equivariant maps, volume 1343 of Lecture Notes in Mathematics. Springer-Verlag, Berlin, 1988.

Noé Bárcenas

Centro de Ciencias Matemáticas

UNAM Campus Morelia Ap. Postal 61-3 Xangari

58089 Morelia, Michoacán, MÉXICO

E-mail: barcenas@matmor.unam.mx

$U R L$ : http://www.matmor.unam.mx/ barcenas 